\title{
STUDY OF BAD BLOCK MANAGEMENT AND WEAR LEVELING IN NAND FLASH MEMORIES
}

\author{
Supriya Kulkarni $\mathbf{P}^{1}$, Jisha $\mathbf{P}^{2}$ \\ ${ }^{I}$ Student, ${ }^{2}$ Assistant Professor, Electronics \& Communication Dept, MVJ College of Engineering, Bangalore, India \\ supriyakul059@gmail.com,jishahaneesh@gmail.com
}

\begin{abstract}
NAND Flash devices have become preferred choice in high density, low cost and high read and write operations where in very large sequential data has to repeatedly written and read at higher rate. However it is hindered by a character called "Lifetime". Typically NAND Flash devices wear out providing around 10,000 to 100,000 life cycles. In this paper we are concerned to discuss about the techniques called Bad-Block Management (BBM) and Wear-leveling to increase the Lifetime of the NAND Flash memories.
\end{abstract}

Keywords: NAND Flash memory, BBM, Wear-leveling, Lifetime.

\section{INTRODUCTION}

FLASH memories are electronic nonvolatile storage devices that can be electrically erased and reprogrammed. There are two types of Flash memories: NOR Flash memory and NAND Flash memory. Embedded systems have traditionally utilized NOR devices. However, high density, low cost and high speed READ/WRITE operations has moved the trend towards NAND Flash device.

\subsection{NOR Versus NAND Technology}

There are specific advantages and disadvantages to in using NAND Flash or NOR Flash in embedded systems. NAND Flash is best suited for file or sequential-data applications; NOR Flash is best suited for random access. The comparison is tabulated in Table1. Even though NAND Flash devices has disadvantages of Slow random access and Byte WRITEs difficult, the real benefits of NAND Flash are faster PROGRAM and ERASE times, as NAND Flash delivers sustained WRITE performance exceeding $5 \mathrm{MB} / \mathrm{s}$. Block erase times are an impressive $2 \mathrm{~ms}$ for NAND Flash compared with 750ms for NOR Flash. Clearly, NAND Flash offers several compelling advantages
Table1. NOR versus NAND flash memories

\begin{tabular}{|c|c|c|}
\hline \multirow{2}{*}{ Advantages } & NAND & NOR \\
\cline { 2 - 3 } & Fast WRITEs & Random access \\
\hline \multirow{2}{*}{ Disadvantages } & $\begin{array}{c}\text { Slow random } \\
\text { access }\end{array}$ & $\begin{array}{c}\text { Byte WRITEs } \\
\text { possible }\end{array}$ \\
\cline { 2 - 4 } & $\begin{array}{c}\text { Byte WRITEs WRITEs } \\
\text { difficult }\end{array}$ & Slow ERASEs \\
\hline \multirow{2}{*}{ Applications } & $\begin{array}{c}\text { File (disk) } \\
\text { applications }\end{array}$ & $\begin{array}{c}\text { Replacement of } \\
\text { EPROM }\end{array}$ \\
\cline { 2 - 3 } & $\begin{array}{c}\text { Voice, data, } \\
\text { video recorder }\end{array}$ & $\begin{array}{c}\text { Execute directly } \\
\text { from nonvolatile } \\
\text { memory }\end{array}$ \\
\hline
\end{tabular}

As the quest continues for lower-power, lighter, more robust products, NAND Flash will prove to be an ideal solution for a wider range of applications. Fig .1 shows how demand for NAND Flash has been driven by three major markets-digital camera media cards, USB flash drives, and MP3 players.

NAND Flash is better suited to meet the storage requirements of many consumer audio and video products, especially lowcapacity applications (4GB or less). 


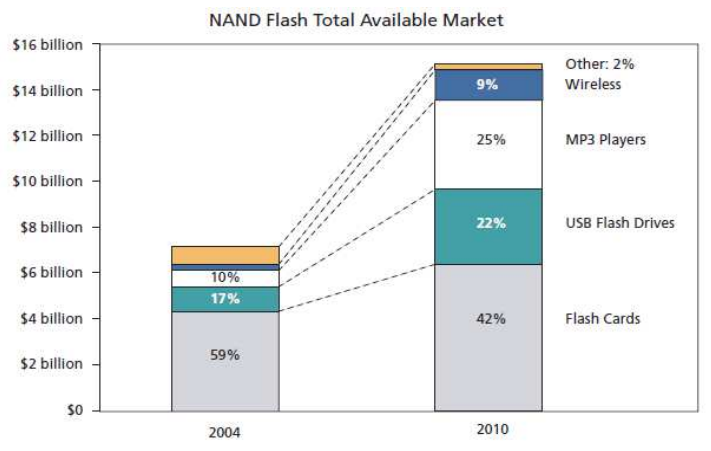

Fig1. Demand for NAND Flash market

\subsection{NAND Flash Architecture}

A typical 2Gb Single Level Cell (SLC) NAND Flash device is organized as 2,048 blocks, with 64 pages per block (fig .2). Each page is 2,112 bytes, consisting of a 2,048-byte data area and a 64-byte spare area.

The spare area is typically used for Error Control and Coding (ECC), wear-leveling, and other software overhead functions, although it is physically the same as the rest of the page. NAND Flash devices are offered with either an 8- or a 16-bit interface. Host data is connected to the NAND Flash memory via an 8-bit- or 16-bit-wide bidirectional data bus.

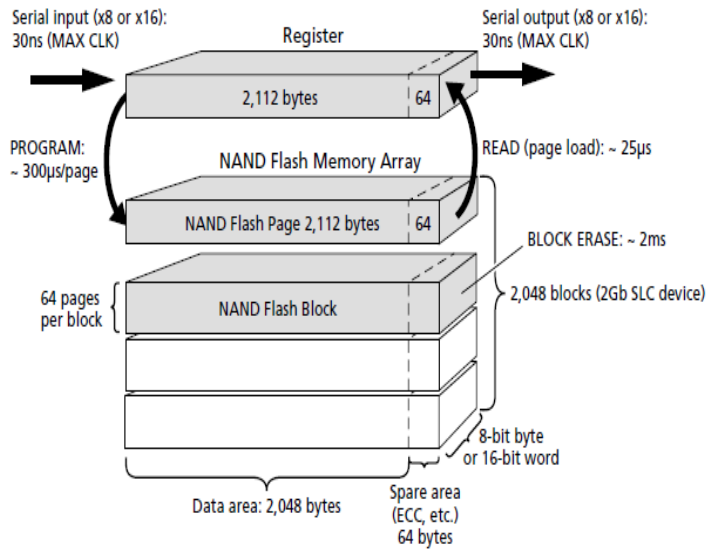

Fig2. NAND Flash Architecture

Erasing a block requires approximately $2 \mathrm{~ms}$. After the data is loaded in the register, programming a page requires approximately $300 \mu \mathrm{s}$. A PAGE READ operation requires approximately $25 \mu \mathrm{s}$, during which the page is accessed from the array and loaded into the 2,112-byte register. The register is then available for the user to clock out the data.

During WRITE operation the data is stored in a unit called "page" and can only be written if it is empty. Therefore if we want to reprogram or write a new data we have to erase the previous one present and rewrite. But ERASE operation in NAND Flash memory is block-by-block so even to reprogram a smaller part of data entire block has to be ERASED. If we want to reuse a page along with such a limitation we have to copy the old data into another (valid) location or page and the entire page can be deleted. This procedure is time consuming and tedious. Instead of directly writing the new data as before the Flash controller has a communication interface called Flash Translation Layer (FTL) which connects the NAND Flash with the Host system. Using this FTL the device maps this Logical address to Physical address through Logical Block Addressing (LBA). When the new data has to be written this LBA will write the new data in the next available location and marks the previous data as "invalid". But this process requires more memory space so in section II and III we discuss optimum algorithm to utilize the entire available space as well as increase the Lifetime of the NAND Flash device.

\subsection{NAND Basics}

NAND Flash memories stores data in array of memory cells made of floating gate (FG) transistors. When a voltage is applied the electrons flow freely between controlled gate (CG) and the channel which is called as floating gate region. To PROGRAM a cell, the voltage is applied at CG, this attracts the free electrons towards electrically isolated FG and the electrons will be trapped in the region beneath $\mathrm{CG}$ (fig .3). Under normal conditions these electrons can store up to several years.



Fig3. Floating gate transistor

Similarly to ERASE a cell, voltage is applied at the opposite side of the channel and CG will be grounded this removes the charges which are present in the FG region. And to check STATUS again high voltage is applied to CG and based on the amount of energy it takes to complete the circuit determines the state of the cell. When such excessive PROGRAM/ERASE conditions are encountered then the trapped charges will be under stress and causes leakage in the oxide and leads to bit failure/error. This is shown in fig .4. 


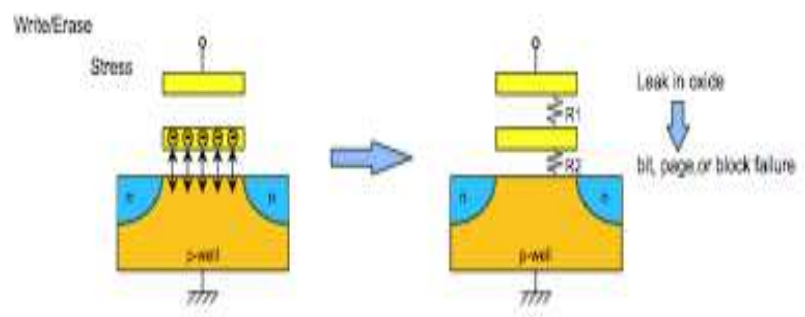

Fig4. Stress and leakage in NAND Flash devices Source: Disturb Testing in Flash Memories

\subsection{Lifetime of Flash Devices}

For systems that have a file allocation table (FAT) based file system, the FAT table is always stored in the same logical blocks. Frequent FAT table updates are required during data a WRITE operation, which implies frequent erase cycles on the same physical blocks, hence a reduced NAND Flash lifetime. The following example calculates how many times a FAT table (cluster size of $2 \mathrm{~KB}$ ) is updated when writing a $10 \mathrm{MB}$ file to a NAND Flash memory with a physical erase unit of 16KB (NAND small page device).

To write a file of $10 \mathrm{MB}, 5 \mathrm{~KB}$ entries in FAT and $5 \mathrm{~KB}$ clusters in the file system are required. This corresponds to 640 physical NAND Flash blocks.

This means that the file can be written at the same location 20 times:

$$
20 \times 5120=102400
$$

This is greater than the maximum number of program/erase cycles.

The expected NAND Flash lifetime can be calculated as follows:

Expected lifetime $=\frac{\text { Size of NAND Flash } \times \text { FAT overhead }}{\text { bytes written per day }}$

This means that if the application writes at $3 \mathrm{~KB} / \mathrm{s}$, the expected lifetime of the NAND blocks is:

$$
\begin{aligned}
\text { Expected lifetime } & =\frac{10 \text { Mbyte } \times 20 \times 0.7}{(3 \text { Kbyte } / \mathrm{s}) \times 24 \times 60 \times 60} \\
& =0.55 \text { days }
\end{aligned}
$$

In a NAND Flash, when logical blocks are mapped to the same physical blocks, the lifetime of the device is significantly reduced, independently of its size. In sections further we shall see different techniques to increase the life span and efficient utilization of the NAND flash memory.

\section{Bad Block Management}

With use, memory cells that forms blocks of the NAND Flash memory array can wear out. Most of the NAND Flash devices contain some initial bad blocks within the memory array. These blocks are typically marked as bad by the manufacturer, indicating that they should not be used in any system. NAND Flash device data sheets provide the location of bad-block markings. Factory testing is performed under worst-case conditions, and those blocks that fail this testing are marked bad. If a factory-marked bad block is used in a system it may appear to operate normally, but may cause other, good blocks to fail, or create additional unforeseen system errors.

\subsection{Recognizing Bad Blocks}

After the original bad-block table is created, if in the time span any other blocks go bad those should also be included in the "invalid block list". In general, for SLC large page (2112byte) devices, any block, where the 1st and 6th bytes/1st word in the spare area of the 1st page, does not contain FFh is a bad block. So new block which come under permanent failure has to place in bad block table, if the error is temporary then can be corrected by Flash controller i.e., if Flash Translation layer addresses one of the Bad Blocks, then Bad Block Management program directs it to a good block. The fig .5 shows the flow chart representing the same.

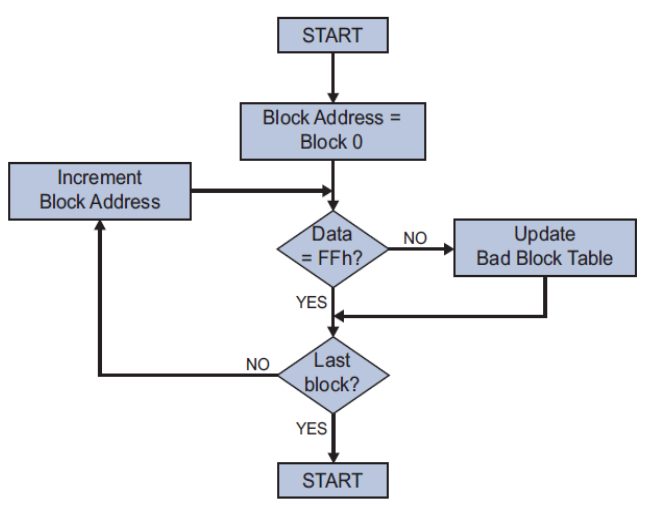

Fig5. Flow chart for recognizing bad blocks

\subsection{Block Replacement}

NAND devices have READ STATUS command after an READ/ERASE operation. This reports a failure in PROGRAM (ERASE) if at least on bit in the programmed (erased) page did not change from " 1 " to a " 0 "state (" 0 " to a "1" state). The additional bad blocks are identified when attempts to program or erase give errors in the status register. As the failure of a page program operation does not affect the data in other pages in the same block, the block can be replaced by reprogramming the current data and copying the rest of the replaced block to an available valid block 


\subsection{Skip Block Method}

In the skip block method the algorithm creates the bad block table and when the target address corresponds to a bad block address, the data is stored in the next good block, skipping the bad block. When a bad block is generated during the lifetime of the NAND Flash device, its data is also stored in the next good block. In this case, the information that indicates which good block corresponds to each developed bad block also has to be stored in the NAND Flash device.

\subsection{Reserve Block Method:}

In the reserve block method, bad blocks are not skipped but replaced by good blocks by redirecting the FTL to a known free good block. For that purpose, the bad block management software creates two areas in the NAND Flash: the user addressable block area and the reserved block area as shown in Fig .6. The FTL can use the user addressable block area to store data whereas the reserved block area is only used for bad block replacement and to save the bad block table that also keeps track of the remapped developed bad blocks.

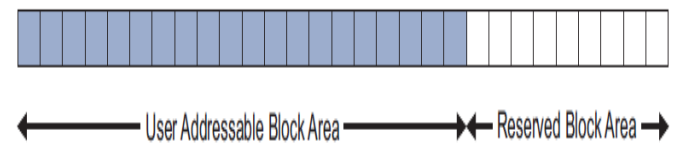

Fig6. Reserve block method

\section{WEAR LEVELING}

In Flash memories, each physical block can be programmed and erased reliably up to 100,000 and 10,000 times, respectively. For write-intensive applications, it is recommended to implement a wear leveling algorithm to monitor and spread the number of write cycles per block. In memory devices where wear leveling is not used their leaves most of the blocks as unused. The wear leveling algorithm ensures that equal use is made of all the available write cycles for each block.

Wear leveling is implemented in FTL. The FTL allows operating systems to read and write to NAND Flash memory devices in the same way as disk drives and maps logical address to physical addresses. Fig .7 shows the wear leveling implemented using FTL. There are two types of wear leveling algorithms implemented in FTL based on Bad Aging Table (BAT): Dynamic wear leveling and Static wear leveling.

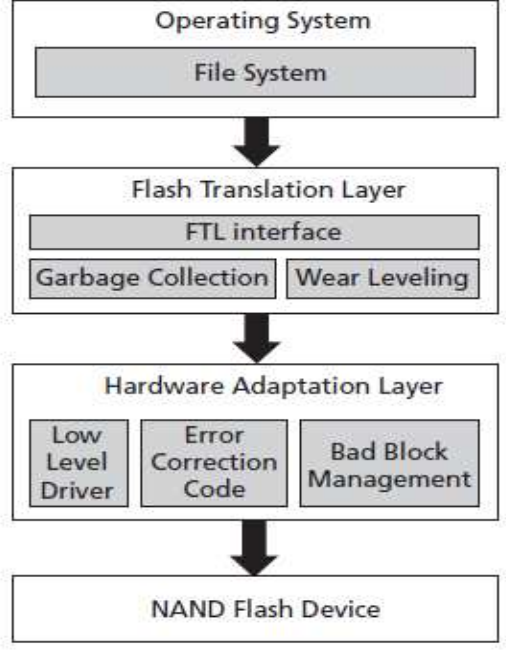

Fig7. Wear leveling in FTL

\subsection{Dynamic Wear Leveling}

When applying the dynamic wear leveling, new data is programmed to the free blocks (among blocks used to store user data) that have had the fewest WRITE/ERASE cycles.

\subsection{Static Wear Leveling}

With static wear leveling, the content of blocks storing static data (as code) is copied to another block so that the original block can be used for data that is changed more frequently. Static wear leveling is triggered when the difference between the maximum and the minimum number of WRITE/ERASE cycles per block reaches a specific threshold. With this particular technique, the mean age of physical NAND blocks is maintained constant.

\subsection{Extended Lifetime}

Wear leveling extends the lifetime of NAND Flash devices because it ensures that even if an application writes to the same logical blocks over and over again, the PROGRAM/ERASE cycles will be distributed evenly over the NAND Flash memory.

For example, the expected lifetime of a $64 \mathrm{MB}(512 \mathrm{Mb})$ NAND Flash device can be calculated as follows:

$$
\begin{aligned}
\text { Expected lifetime } & =\frac{64 \text { Mbyte } \times 100 \text { Kcycles } \times 0.7}{(3 \text { Kbyte } / \mathrm{s}) \times 24 \times 60 \times 60} \\
& =18,124 \text { days }(\text { about } 49.7 \text { years })
\end{aligned}
$$

In this example, 0.7 is the file system overhead, which shows that implementation of algorithms, has efficiently increased the lifetime from 0.55 days up to 49.7 years. 


\section{CONCLUSIONS}

The algorithms discussed here can help designers more fully exploit the capabilities of NAND Flash devices, ensure consistent data integrity, and deliver better performance in their embedded systems. It is proved that implementation of bad block management algorithms with error correction code (ECC) algorithms and wear leveling as part of the software tool chain has increased the lifetime of NAND Flash in an embedded system.

\section{REFERENCES}

[1] TN-29-19: NAND Flash 101 Introduction, "An Introduction to NAND Flash and How to Design It In to Your Next Product"@2006 Micron Technology, Inc.

[2] TN-29-17: NAND Flash Design and Use Considerations Introduction, "Technical Note Design and Use Considerations for NAND Flash Memory”, (C2006 Micron Technology, Inc.

[3] TN-29-61: Wear Leveling in NAND Flash Memory

Introduction, "Technical Note Wear Leveling in Micron ${ }^{\circledR}$ NAND Flash Memory", (C2006 Micron Technology, Inc.

[4] AN1819 APPLICATION NOTE," Bad Block Management in NAND Flash Memories", (C) 2004 STMicroelectronics GROUP OF COMPANIES

[5] Error Detection/Correction And Bad Block Management, "Early Detection of Factory-Marked Bad Blocks And Normal Operation Tracking Of Bad Blocks in Solid-State Drives (SSDs), An InnoDisk White Paper, August 2012,

[6] Ken Whitaker, "A Comparative Study of Flash Storage Technology for Embedded Devices”, (C) 2006-2012 Datalight, Inc

[7] NAND Basics, "Understanding the Technology Behind Your SSD", www.samsung.com

[8] Understanding SSDs, "A Peek Behind the Curtain", www.samsung.com.

[9] Rino Micheloni, "Non-Volatile Memories for Removable Media", IEEE proceedings, Vol. 97, No. 1, January 2009, pp.148-160

[10] Douglas Sheldon and Michael Freie, "Disturb Testing in Flash Memories", Jet Propulsion Laboratory California Institute of Technology Pasadena, California, JPL Publication 08-7 3/08

[11]. Chundong Wang and Weng-Fai Wong, "Extending the Lifetime of NAND Flash Memory by Salvaging Bad Blocks", School of Computing, National University of Singapore, Singapore

[12] Flash memory, www.wikipedia.com. 